\title{
Preparation of porous carbon particle with shell/core structure
}

\author{
Y. B. Jiang ${ }^{1,2}$, L. H. Wei ${ }^{3}$, Y. Z. Yu ${ }^{1}$, T. Zhao ${ }^{*}$ \\ ${ }^{1}$ Beijing National Laboratory for Molecular Sciences, Laboratory of Advanced Polymer Materials, Institute of Chemistry, \\ Chinese Academy of Sciences, Beijing 100080, P. R. China \\ ${ }^{2}$ Graduate University of Chinese Academy of Sciences, Beijing 100039, P. R. China \\ ${ }^{3}$ Department of Chemistry, Zhengzhou University, Zhengzhou, 450001, P. R. China
}

Received 12 March 2007; accepted in revised form 19 April 2007

\begin{abstract}
Porous carbon particles with a shell/core structure have been prepared successfully by controlled precipitation of the polymer from droplets of oil-in-water emulsion, followed by curing and carbonization. The droplets of the oil phase are composed of phenolic resin (PFR), a good solvent (ethyl acetate) and porogen (Poly(methyl methacrylate), PMMA). The microstructure was characterized in detail by scanning electron microscopy (SEM), transmission electron microscopy (TEM), nitrogen adsorption, and thermo gravimetric analysis (TGA). The obtained carbon particles have a capsular structure with a microporous carbon shell and a mesoporous carbon core. The BET surface area and porous volume are calculated to be $499 \mathrm{~m}^{2} \mathrm{~g}^{-1}$ and $0.56 \mathrm{~cm}^{3} \mathrm{~g}^{-1}$, respectively. The effects of the amount of porogen (PMMA), co-solvent (acetone) and surfactant on the resultant structure were studied in detail.
\end{abstract}

Keywords: polymer composites, phase separation, polymer blend, porous carbon

\section{Introduction}

In recent years, there has been growing interest in new applications of porous carbons because of their characteristic physicochemical properties, such as, their large surface area, large pore volume, thermal and chemical stability. Porous carbon particles are of great interest in column packing, filler, hydrogen storage, supercapacitors, catalyst supports and other applications [1].

Several methods have been developed to prepare spherical carbon [2-8]. The solid-templating approach has been widely used for the synthesis of a variety of nanoporous carbon materials [5-9]. Although precise control of pore size and pore structures is desirable, it has several limitations such as incomplete and time consuming infiltration of the precursor, laboring and expensive template removal process. The commercially available and inexpensive Pluronic block copolymers have been used as templates to prepare mesoporous carbon with various pore structures $[10,11]$, but direct synthesis of porous carbon particles still remains challenging by this method. Polymer as porogen to prepare porous carbon is another efficient method. Yamazaki et al prepared nanostructure carbonaceous material with continuous pores from reaction-induced phase separation of miscible polymer blends $[12,13]$. As PFR interacts strongly with PMMA via hydrogen bonds, the miscibility of PMMA to PFR is perfect, and the phase separation occurs after the curing reaction has advanced to some extent. After PMMA removal and carbonization, the carbonaceous materials with continuous pores can be obtained. Furthermore, porous carbon materials having hierarchically structures have

*Corresponding author, e-mail: tzhao@iccas.ac.cn

(c) BME-PT and GTE 


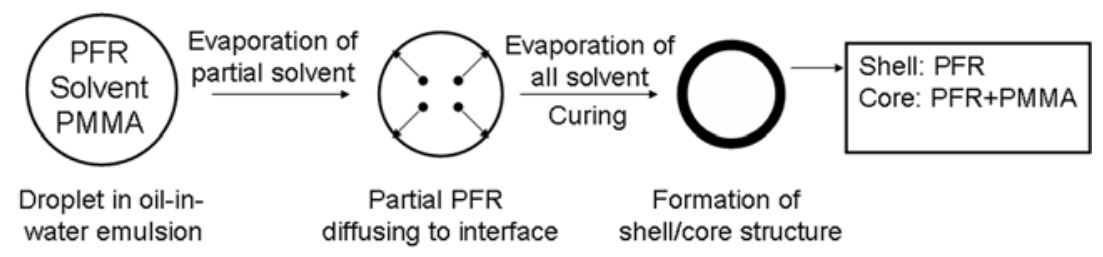

Figure 1. Schematic illustration for capsular structure formation

attracted much interest because of their special character $[2,5,8]$.

In this paper, we prepared carbon particles with a microporous carbon shell and a mesoporous carbon core through the method called 'internal phase separation' within the droplets of an oil-in-water emulsion. This method has been used in several reported works. Mathiowitz et al prepared double-walled microspheres by this one-pot method for controlled drug release [14, 15]. Vincent et al prepared a series of polymer microcapsules with liquid cores also by this method [16-18]. In our work, PFR has been used as the shell material, and PMMA as the porogen. As described by Solomon et al, the PMMA domain size in the blends (PFR/PMMA) before curing was 2 to $3 \mathrm{~nm}$ and it became 20 to $30 \mathrm{~nm}$ after full curing [19]. The process for the formation of the shell/core structure is illustrated in Figure 1. The organic phase containing PFR and PMMA was emulsified in the aqueous surfactant. After solvent removal, hydrophilic PFR preferred to diffuse to the interface of oil-water. But because of good miscibility of PFR and PMMA, partial PFR retained to blend with PMMA and the capsule structure formed with PFR shell and blends of PFR/PMMA core. After carbonization, the final products with microporous carbon shells and mesoporous carbon cores were prepared. The carbon materials with special structures in our work will show advantages in many fields such as controlling release, hydrogen storage, electrochemical double-layer capacitors and so on.

\section{Experiment}

\subsection{Materials}

Phenol, formaldehyde (37\% aqueous solution) and hexamethylenetetramine were purchased from Beijing Yili Chemicals Factory and used to synthesize phenolic resin. Poly(methyl methacrylate) (PMMA, Aldrich, $\left.M_{w}=10,000\right)$ was used as the porogen. Gum arabic and sodium dodecyl sulfate (SDS, Tianjin Kemiou Chemical Regents Factory, China) were used as emulsifiers. The solvents used were supplied by Beijing Chemicals Factory, China. All the reagents were of analytical grade and used as received. Doubly deionized water was used as the aqueous medium.

\subsection{Synthesis of phenolic resin}

The PFR used as the carbon precursor was synthesized and purified as described by Brode [20]. In a typical synthesis, the mixture of phenol $(50 \mathrm{~g})$, aqueous formaldehyde $(50 \mathrm{~g})$, hexamethylenetetramine $(4.5 \mathrm{~g})$, gum arabic $(0.5 \mathrm{~g})$ and water $(27.5 \mathrm{~g})$ was charged into a three-necked flask equipped with a mechanical stirrer, a thermometer and reflux condenser. The reaction temperature was maintained at $85^{\circ} \mathrm{C}$ in a water bath for $70 \mathrm{~min}$. The obtained particulate resol was collected by filtration, washed with distilled water for three times and dried at $40^{\circ} \mathrm{C}$ for $2 \mathrm{~h}$.

\subsection{Preparation of porous carbon}

PFR was dissolved in the mixture of ethyl acetate and acetone, and then PMMA was added. Aqueous surfactant solution was charged into a three-necked round-bottomed flask equipped with a mechanical stirrer. The organic solution was added to the aqueous surfactant solution, and the mixture was emulsified mechanically at a rate of $3000 \mathrm{rpm}$ to form an oil-in-water emulsion. After agitation lasting for $1 \mathrm{~h}$ at $25^{\circ} \mathrm{C}$, the diluted emulsion was evaporated for 40 min at $30^{\circ} \mathrm{C}$ under reduced pressure, followed by transferring into a Teflon-lined autoclave, treated at $130^{\circ} \mathrm{C}$ for about $6 \mathrm{~h}$ and another $6 \mathrm{~h}$ at $150^{\circ} \mathrm{C}$, then allowed to cool down to room temperature gradually. The resultant microsphere dispersion was collected with centrifugation, and then washed with distilled water, ethanol, and ethyl acetate, finally dried in vacuum oven for $10 \mathrm{~h}$ at $60^{\circ} \mathrm{C}$. The solid sample obtained was carbonized under a nitrogen atmosphere at $800^{\circ} \mathrm{C}$ for 4 hours at a heating rate of $1^{\circ} \mathrm{C} \mathrm{min}^{-1}$ to obtain porous carbon materials. 


\subsection{Characterization}

SEM Scanning electron microscopy (SEM, HITACHI S-4300, Japan) was used to investigate the morphology of the products. One drop of the microsphere dispersion was placed on a stainless steel SEM stub and allowed to air-dry overnight. Before observation, the dried sample was coated with a layer of gold with a thickness of approximately $10 \mathrm{~nm}$.

TEM Transmission electron microscopy (TEM) pictures were taken on a JEOL 2010 microscope (Japan) with an acceleration voltage of $200 \mathrm{kV}$. Samples for TEM measurements were diluted and mounted on 400-mesh carbon-coated copper grids and left to dry at room temperature.

Nitrogen sorption Nitrogen sorption was performed on a Micromeritics ASAP 2020M Porosity Analyzer. The samples were degassed at $250^{\circ} \mathrm{C}$ for $6 \mathrm{~h}$ before the measurement. The specific surface area was determined by the Brunauer-EmmettTeller (BET) method. The BJH (Barret-Joyner-Halenda) method was applied to analyze the mesopore size distributions using the adsorption branch.

TGA Thermo gravimetric analysis (TGA, Netzsch STA409pc) was employed to monitor the degradation behavior of as-prepared products. The sample was heated at a heating rate of $10^{\circ} \mathrm{C} \mathrm{min}^{-1}$ in $\mathrm{N}_{2}$ atmosphere.

\section{Results and discussion}

\subsection{Characterization of porous carbon particles}

TGA analysis was employed to monitor the degradation behavior of PFR, PMMA and as-prepared blending microsphere. Notable weight loss of approximately $99 \%$ (Figure 2) is observed in the temperature range of $300 \sim 400^{\circ} \mathrm{C}$ for pure PMMA, suggesting that most of the templates can be decomposed under nitrogen before $400^{\circ} \mathrm{C}$. Compared with the TGA curve of PFR, the blending microsphere has a lower mass residue, which is mainly attributed to the degradation of PMMA. Therefore, simple heating treatment can be utilized to efficiently decompose and remove the porogen (PMMA) while generating porosity and maintaining an open framework.

Figure 3 shows representative SEM images of asprepared microsphere and corresponding porous carbon particles. As seen in the images, the poly-

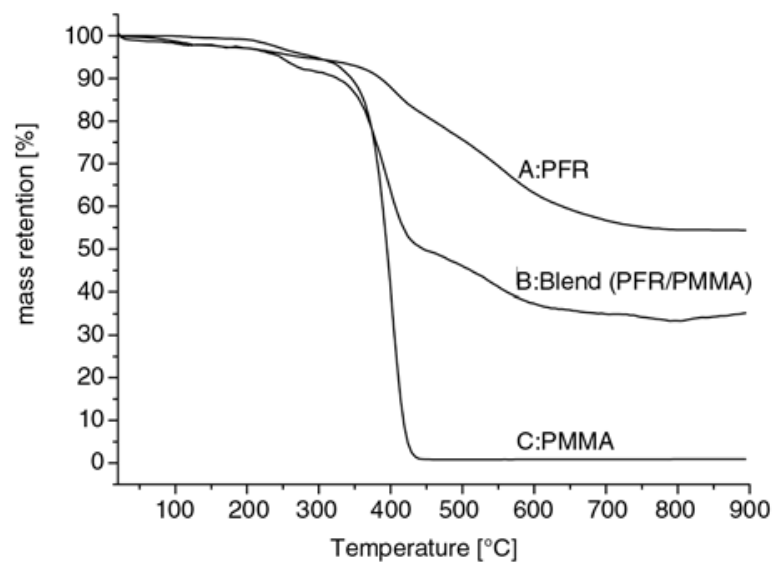

Figure 2. TGA thermograms of PFR, PMMA and blending microspheres. The composition of PFR/PMMA blends was 63/37 in weight

mer and carbon particles are spherical in morphology with polydisperse particles sizes that range from less than $100 \mathrm{~nm}$ to greater than 1um. Figure 3a shows the composite particles prepared from the PFR/PMMA blend (63/37 in weight). These microspheres have smooth surface with no pores or defects. After carbonization, as shown in Figure 3b, the obtained carbon particles can be observed shrinking on the surface, but still with no visible pores or defects. When carbon particles were fractured (shown in Figure 3c and d), obvious shell/ core structure was observed. Small carbon particles interconnected to form the mesoporous structure surrounded by the microporous carbon shell. The formation of the capsule structure can be attributed to the two phase separation processed: solvent evaporation induced phase separation and curing induced phase separation. As shown in Figure 1, after solvent removal, hydrophilic PFR diffused to the interface of oil-water. But because of good miscibility of PFR and PMMA, the capsule structure was formed with a PFR shell and blends of PFR/ PMMA core. After curing, phase separation occurred in the core, and PFR shell was preserved. Therefore, after PMMA removal and carbonization, the final structure was obtained.

Nitrogen adsorption-desorption isotherms and the resulting pore size distribution are shown in Figure 4 . The isotherms are similar to type IV isotherms with a hysteresis loop and a steep adsorption step at a relative pressure of 0.9 . The non-parallel hysteresis reflects a non-uniform pore connectivity that is due to the existence of some larger pores. The resulting pore size distribution displays that most of mesopore is about $4 \mathrm{~nm}$ and a small quantity of 

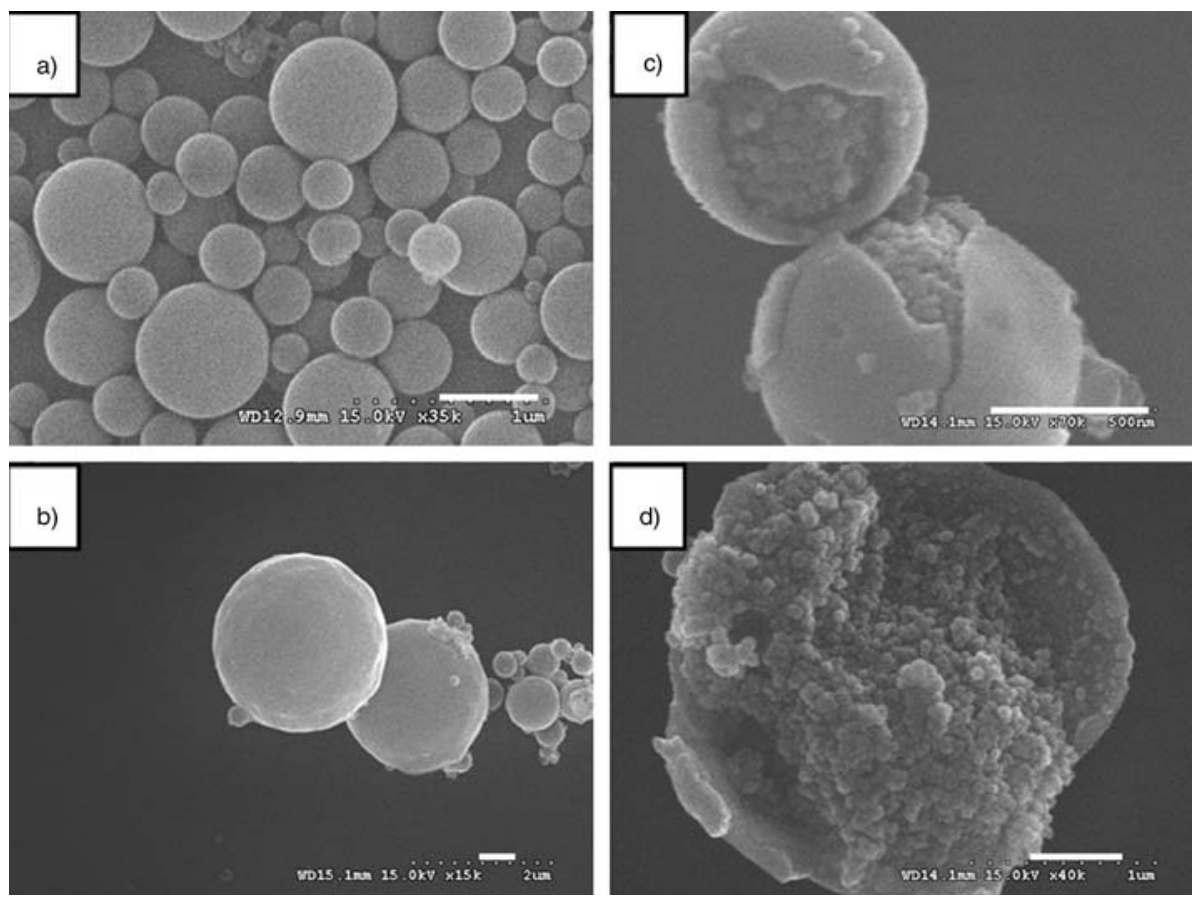

Figure 3. SEM images of as-prepared PFR/PMMA blending microspheres (a) and carbon particles (b, c, d). The composition of PFR/PMMA blends was 63/37 in weight. The scale bar is $500 \mathrm{~nm}$

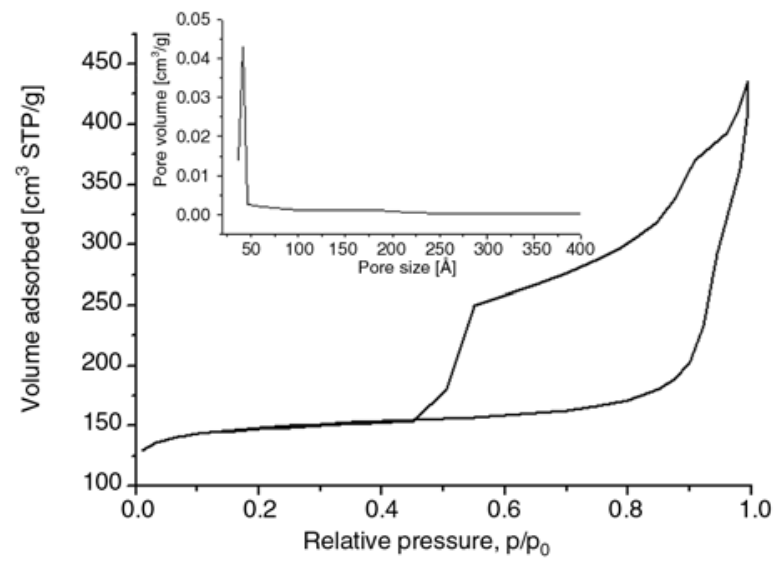

Figure 4. Nitrogen adsorption/desorption isotherms and pore size distribution (insets) for carbon particles. The composition of PFR/PMMA blends was $63 / 37$ in weight

larger pores less than $25 \mathrm{~nm}$ exist, which agrees with the desorption isotherm in Figure 4. The calculated BET surface area and pore volume of the carbon particles is $499 \mathrm{~m}^{2} \mathrm{~g}^{-1}$ and $0.56 \mathrm{~cm}^{3} \mathrm{~g}^{-1}$, respectively.

\subsection{Effect of the composition of phenolic resin/PMMA blends}

The component of blends (PFR/PMMA) is the most important factor to the final structure of carbon particles. Figure 3 shows the images of carbon with low PMMA content. But when increasing the
PMMA content (50/50) as shown in Figure 5, the similar structure as shown in Figure 3 is formed, but the core size is smaller than that of the shell, and the hollow carbon sphere with a movable porous carbon core is obtained. Furthermore, when the amount of PMMA increased to 37/63, no spherical morphology is observed. Only nanosheet and small carbon particles are contained in the SEM images. From these fragments, we conclude the formation of a shell-core structure. However because of the thinner shell wall and smaller core, the strength of the shell is not enough to maintain the spherical shape. The results can be explained from the phase separation by solvent evaporation. When the amount of PMMA increased, both the amount of PFR diffusing to the oil-water interface, and that remaining in the core, decreased. Carbonization removed all the PMMA and about 50\% of the PFR, the large amount of mass loss of the composite finally induced structure collapse.

\subsection{Effect of co-solvent content}

Ethyl acetate was used as the solvent to prepare the materials. However, a weak polarity makes it an unsuitable solvent for PFR. Acetone was added as the co-solvent. We studied the effect of the amount of acetone on the structure of final carbon particles. Figure 6 shows the SEM images of carbon particles 

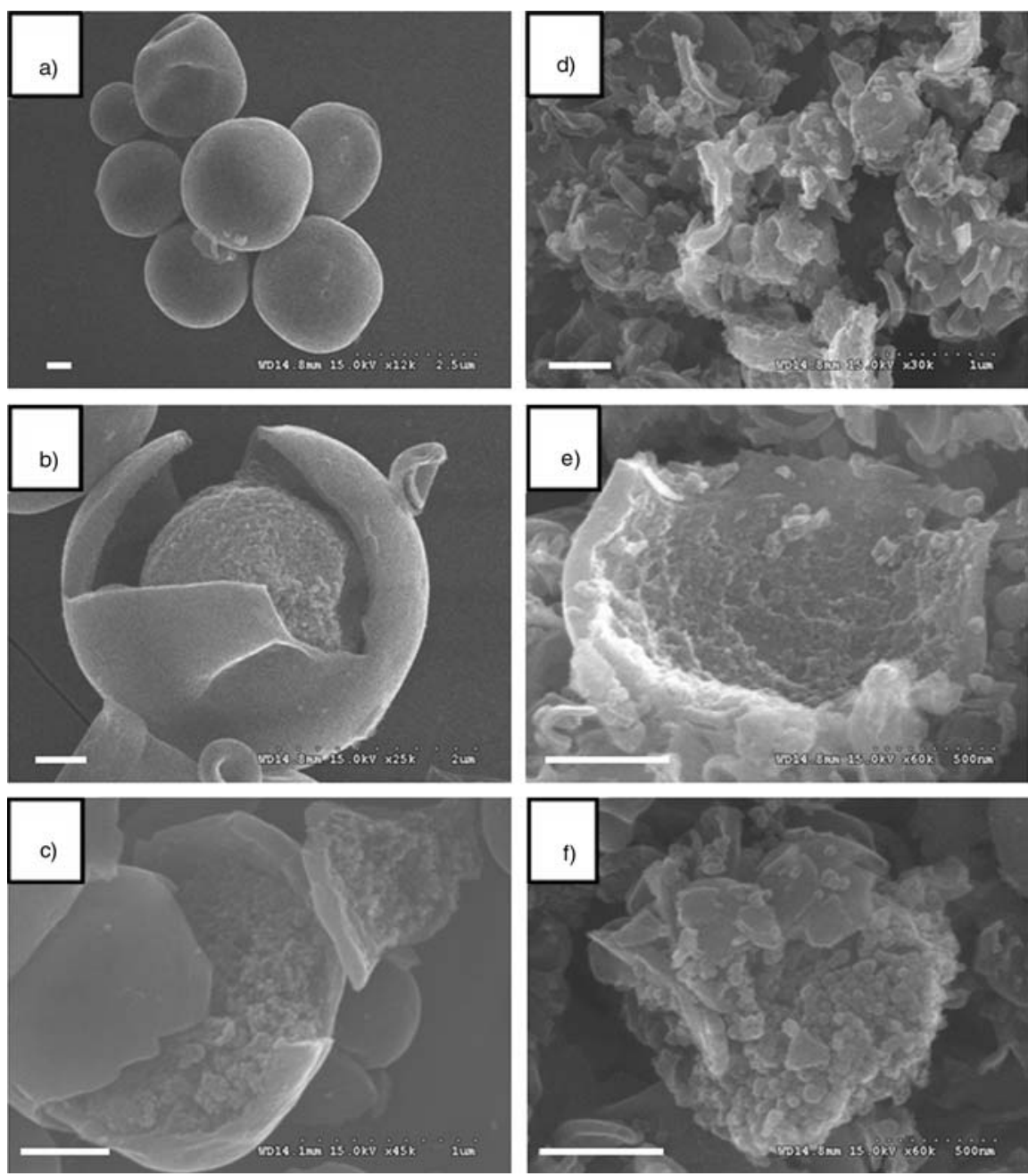

Figure 5. SEM images of obtained carbon with different composition of PFR/PMMA blends in weight. a, b, c: 50/50; $\mathrm{d}$, e, f: $37 / 63$. The scale bar is $500 \mathrm{~nm}$

prepared with different amounts of acetone added. With the increase of amounts of acetone, the distribution of particle size narrowed, and the average particle diameter increased obviously (Figure 6a, b). But with the further increase of acetone, the distribution of particle size widened. These results suggest that the content of acetone can affect the emulsification process, and therefore affect the final particle morphology. This is consistent with the reported results [16].

\subsection{Effect of surfactant content}

The structure shown in Figure 3 was prepared from the solution emulsified by $2 \%$ gum Arabic and $0.1 \%$ SDS as emulsifiers. When changing the emulsifiers' content to $2 \%$ gum Arabic and $0.2 \%$ SDS, the structure obtained changed much. As shown in Figure 7, the as-prepared polymer microcapsule has a hollow cavity, and there is a narrow channel in the shell wall. The TGA curves and residual mass of the product is similar to that of pure PFR (not shown here), it suggests that the component of the hollow sphere is PFR. The formation of the peculiar structure may result from increasing the surfactant. It decreased the oil/water interfacial tension too much, which would decrease the driving force of polymer diffusion to the oil/water interface. This will lead to the result that the region where the adsorption of polymer is insufficient would be occupied by the surfactant molecular aggregates. After curing and washing with the solvent, PMMA escaped from the core, and was removed by the solvent. Finally, a cavity and a channel were left. Further study on the formation mechanism and application potential are in progress.

\section{Conclusions}

Porous carbon particles have been prepared by the method of polymer blend and internal phase sepa- 

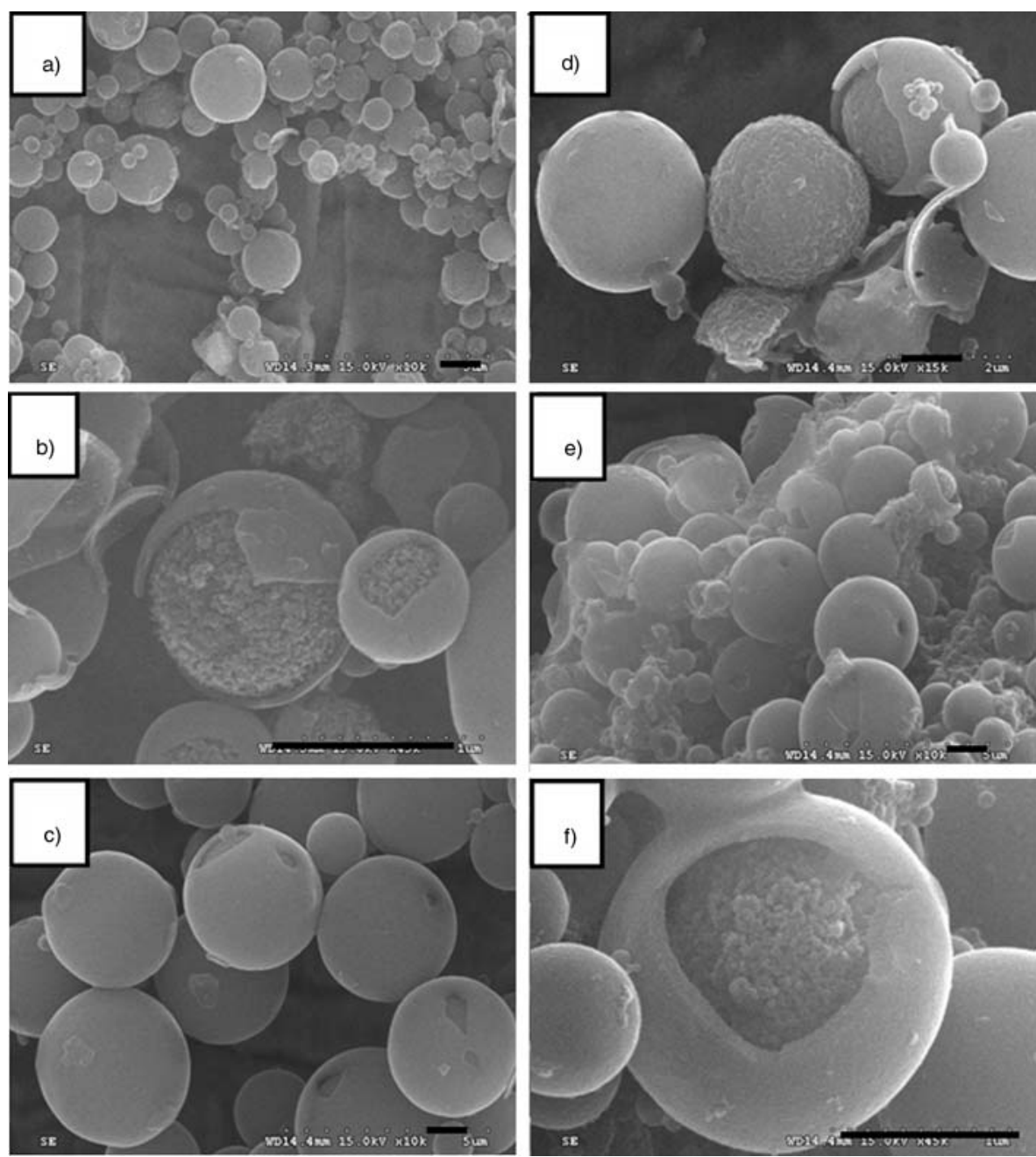

Figure 6. SEM images of obtained carbon with different content of acetone added. a, b: 20\%; c, d: $33 \%$; e, f: $50 \%$. The scale bar is $1 \mu \mathrm{m}$
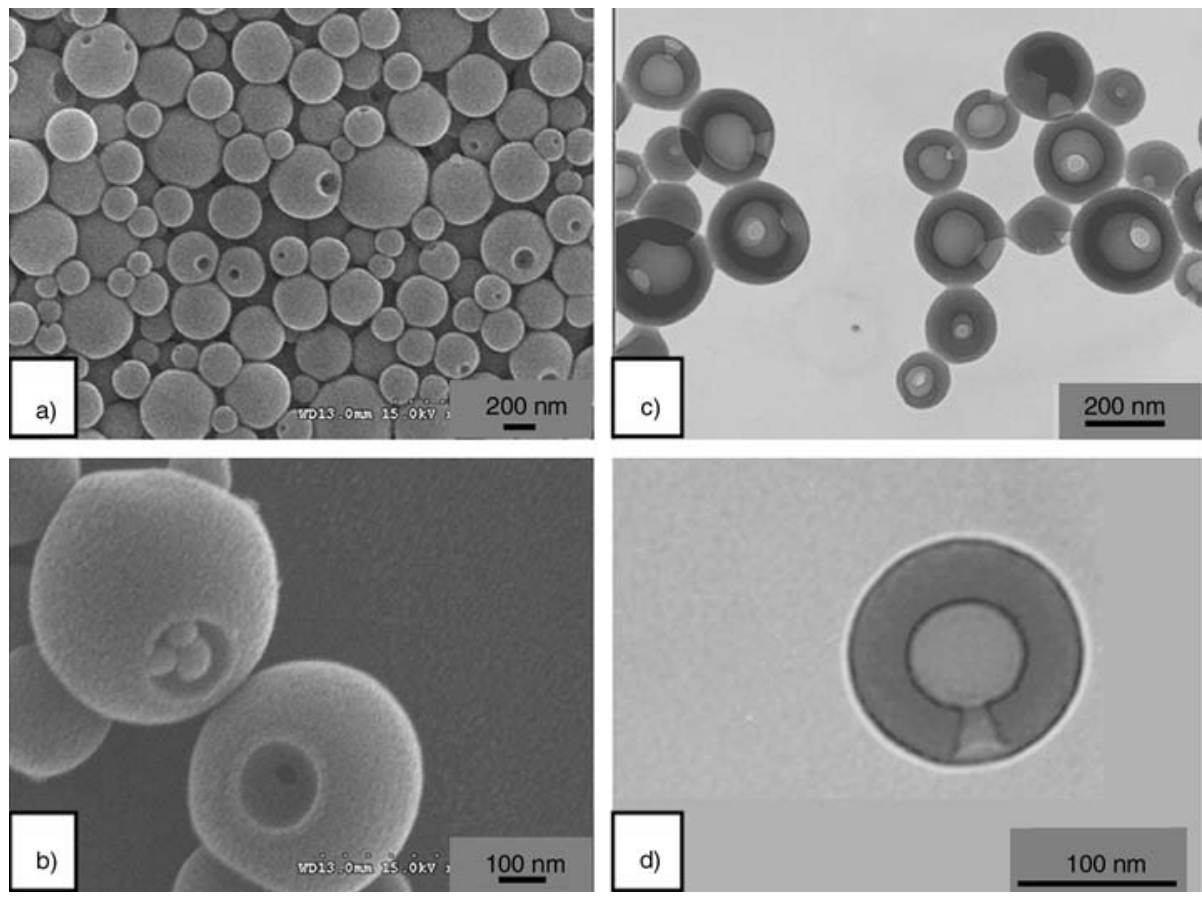

Figure 7. SEM (a, b) and TEM (c, d) images of obtained hollow carbon particles with one channel on the shell 
ration. PFR was used to serve as the carbon precursor and PMMA as porogen. Through emulsification and solvent removal, the capsular structure was obtained with PFR as the shell and the blend of PFR/PMMA as core. After heat treatment in nitrogen, porous carbon particles with microporous carbon shells and mesoporous carbon cores were formed. The content of PMMA affected the microstructure and the mechanical strength of the resultant carbon materials. The addition of a co-solvent can affect the emulsification process, and appropriate acetone amount can produce carbon particles with narrow size distributions. Varying the surfactant amounts, hollow PFR microspheres with one channel on the shell can be prepared. We believe that carbon materials in our work may find potential applications in such areas as column packing, hydrogen storage and electrochemical double-layer capacitors, etc.

\section{References}

[1] Lee J., Kim J., Hyeon T.: Recent progress in the synthesis of porous carbon materials. Advanced Materials, 18, 2073-2094 (2006).

[2] Hampsey J. E., Hu Q. Y., Rice L., Pang J. B., Wu Z. W., Lu Y. F.: A general approach towards hierarchical porous carbon particles. Chemistry Communications, (29), 3606-3608 (2005).

[3] Fujikawa D., Uota M., Yoshimura T., Sakai G., Kijima T.: Surfactant-templated synthesis of resorcinol-formaldehyde polymer and carbon nanocomposite: nanospheres and nanowires. Chemistry Letters, 35, 432-433 (2006).

[4] Xia Y. D., Mokaya R.: Ordered mesoporous carbon hollow spheres nanocast using mesoporous silica via chemical vapor deposition. Advanced Materials, 16, 886-891 (2004).

[5] Arnal P. M., Schüth F., Kleitz F.: A versatile method for the production of monodisperse spherical particles and hollow particles: templating from binary coreshell structures. Chemistry Communications, (11), 1203-1205 (2006)

[6] Hampsey J. E., Hu Q. Y., Wu Z. W., Rice L., Pang J. B., Lu Y. F.: Templating synthesis of ordered mesoporous carbon particles. Carbon, 43, 2977-2982 (2005).

[7] Yang M., Ma J., Ding S. J., Meng Z. K., Liu J. G., Zhao T., Mao L. Q., Shi Y., Jin X. G., Lu Y. F., Yang Z. Z.: Phenolic resin and derived carbon hollow spheres. Macromolecular Chemistry and Physics, 207, 1633-1639 (2006).
[8] Yoon S. B., Sohn K., Kim J. Y., Shin C. H., Yu J. S., Hyeon T.: Fabrication of carbon capsules with hollow macroporous core/mesoporous shell structures. Advanced Materials, 14, 19-21 (2002).

[9] Kamil P. G., Mietek J.: Carbons with extremely large volume of uniform mesopores synthesized by carbonization of phenolic resin film formed on colloidal silica template. Journal of the American Chemical Society, 128, 10026-10027 (2006).

[10] Liang C. D., Dai S.: Synthesis of mesoporous carbon materials via enhanced hydrogen-bonding interaction. Journal of the American Chemical Society, 128, 5316-5317 (2006).

[11] Meng Y., Gu D., Zhang F. Q., Shi Y. F., Cheng L., Feng D., Wu Z. X., Chen Z. X., Wan Y., Stein A., Zhao D. Y.: A family of highly ordered mesoporous polymer resin and carbon structures from organicorganic self-assembly. Chemistry of Materials, $\mathbf{1 8}$ 4447-4464 (2006).

[12] Yamazaki M., Teduka M., Ikeda K., Ichihara S.: Preparation of carbon materials with coral-like continuous pores using miscible polymer blends. Journal of Materials Chemistry, 13, 975-977 (2003).

[13] Yamazaki M., Kayama M., Ikeda K., Alii T., Ichihara S.: Nanostructured carbonaceous material with continuous pores obtained from reaction-induced phase separation of miscible polymer blends. Carbon, 42, 16411649 (2004)

[14] Pekarek K. J., Jacob J. S., Mathiowiz E.: Doublewalled polymer microspheres for controlled drug release. Nature, 367, 258-260 (1994).

[15] Pekarek K. J., Jacob J. S., Mathiowiz E.: One-step preparation of double-walled microspheres. Advanced Materials, 6, 684-687 (1994).

[16] Loxley A., Vincent B.: Preparation of poly(methylmethacrylate) microcapsules with liquid cores. Journal of Colloid and Interface Science, 208, 49-62 (1998).

[17] Atkin R., Davies P., Hardy J., Vincent B.: Preparation of aqueous core/polymer shell microcapsules by internal phase separation. Macromolecules, 37, 7979-7985 (2004).

[18] Dowding P. J., Atkin R., Vincent B., Bouillot P.: Oil core-polymer shell microcapsules prepared by internal phase separation from emulsion droplets. I. characterization and release rates for microcapsules with polystyrene shells. Langmuir, 20, 11374-11379 (2004).

[19] Zhang X., Solomon D. H.: Phase structures of hexamine cross-linked novolac blends. 1. blends with poly(methyl methacrylate). Macromolecules, 27, 4919-4926 (1994).

[20] Brode G. L., Kopf P. W., Chow S. W.: Phenolic thermospheres: Chemical design and principles. Industrial Engineering Chemistry Products Research and Development, 21, 142-145 (1982). 